\title{
Intrauterine device insertions: which variables matter?
}

\author{
Windy Mary Brown, ${ }^{1}$ Konia Trouton ${ }^{2}$
}

\begin{abstract}
${ }^{1}$ Family Physician, Station Fort Langley, Langley, British Columbia, Canada

${ }^{2}$ Clinical Associate Professor, Department of Family Practice, Faculty of Medicine, University of British Columbia, Vancouver Island Women's Clinic, Vancouver, British Columbia, Canada
\end{abstract}

\section{Correspondence to Dr Windy M Brown, Station Fort Langley, PO Box 99, Langley, British Columbia, Canada, V1M 2R4; windy.brown@gmail.com}

Received 9 May 2012 Revised 13 February 2013 Accepted 5 March 2013 Published Online First 15 June 2013
To cite: Brown WM, Trouton K. J Fam Plann Reprod Health Care 2014;40:117-121.

\begin{abstract}
Objectives Many factors are suspected to influence the intrauterine device (IUD) insertion process. This study sought to examine the effects of a few key variables on IUD insertion tolerability, complications and follow-up issues.

Methods A retrospective chart review was undertaken of all IUD insertions over an 11-month period at an urban Canadian women's clinic. Linear regression analysis of 354 insertions assessed the effects of parity, age, local anaesthesia and other variables on insertion pain. Chi square $\left(\chi^{2}\right)$ and analysis of variance statistics were employed to evaluate differences in insertion complications by parity and age. A $\chi^{2}$ test was performed to compare follow-up expulsion or removal rates by parity.
\end{abstract}

Results Statistically significant differences were found, with nulliparous women and those who received local anaesthesia reporting more insertion pain. Age, IUD type and recent abortion status did not affect insertion pain. Nulliparous women did not experience significantly more insertion difficulty or complications, nor did they have higher rates of expulsion or removal at follow-up.

Conclusions These findings suggest that the practice of providing cervical anaesthesia at IUD insertion may cause slightly more pain, without any obvious additional benefit. The difference in insertion pain by parity, although statistically significant, is small enough to be of questionable clinical importance. Overall, these findings add to the growing body of evidence for IUDs being safe and well-tolerated in nulliparous women.

\section{INTRODUCTION}

The popularity of intrauterine devices (IUDs) in many areas of the world is increasing, as evidence has now clearly demonstrated that their purported safety risks are largely unfounded. There is good research to show that IUDs do not increase the risk of ectopic pregnancies, nor of pelvic infections beyond the first month after insertion. ${ }^{1-3}$ Although the side effect profiles of IUD use have also

\section{Key message points}

Although nulliparous women had significantly more pain than parous women at intrauterine device (IUD) insertion, the difference is small and unlikely to affect clinical practice.

- Injection of intracervical lidocaine at IUD insertion caused significantly greater pain than no anaesthesia.

- Nulliparous women did not experience significantly more insertion difficulty or complications, nor did they have higher rates of expulsion or removal at follow-up.

been well characterised, the insertion process itself has been less studied. Many potential users ask how painful insertion is and whether or not an IUD is a good choice at their point in the reproductive life cycle. Many clinicians have restrictive notions as to whom an IUD would suit.

Classically, IUDs have been reserved for parous women. Current practice recommends them for nulliparous woman as well, and several recent studies have highlighted their safety and efficacy in that group. ${ }^{4-6}$ However, the effects of parity on insertion tolerability, failure and follow-up complications remain unclear. Some studies report increased insertion discomfort, insertion failure or higher rates of expulsion and discontinuation in nulliparous women whereas others do not. $^{7-14}$ For instance, a large international data set analysis found that vasovagal reactions, although rare, were closely related to moderate or severe insertion pain and were significantly more likely in nulliparous compared to parous women. ${ }^{15}$

Cervical local anaesthetic block might also affect insertion tolerability. Standard procedure for some physicians includes intracervical injection of lidocaine before 
IUD insertion. One small study found that this method reduced insertion discomfort. ${ }^{16}$ Age may also play a role as it has been documented that cervical problems at insertion increase significantly with women's age. $^{17}$

The goal of this study was to assess the effects of parity, local anaesthesia, IUD type and age on IUD insertion tolerability, as well as the effects of parity on post-insertion complications.

\section{METHODS}

A retrospective chart review was performed of IUD insertions from January to November 2007 at an urban women's health clinic run by family physicians in British Columbia, Canada. The specific clinic was chosen because of its high insertion volume, which allowed for assessment of some low-frequency IUD insertion-related complications, and its standardised, thorough method of recording patient encounters. Within a few minutes of IUD insertion, all patients were asked to verbally rate insertion pain on a scale of $0-10$, with 10 being equivalent to the worst pain possible. We defined this number as IUD insertion tolerability. Paper notes at the clinic were ordered sequentially numerically and filed by date. Clinic patients were self- or physician-referred for a variety of reproductive health services including therapeutic abortions. The inclusion criterion was any chart colour-coded as an IUD insertion from 1 January 2007 onwards. The file review start date was chosen such that at least 6 months had lapsed from IUD insertion to review. Exclusion criteria for regression and adverse insertion event analyses were: missing data points for the main criteria of parity or local anaesthetic, missing pain rating, or IUD inserted at the time of therapeutic abortion. For the purpose of follow-up analysis, charts missing pain rating or local anaesthetic data were included.

A minimum sample size of 91 was calculated to give a power level of 0.8 , presuming by convention a medium effect size $(0.15)$ of our explanatory variables on insertion tolerability in a five-factor multiple regression analysis ( $\alpha$ level 0.05 ). Because of our interest in uncommon complications after IUD insertion, we went on to collect as much data as was possible by our chart reviewers. Post hoc power analysis in fact showed a power level of 0.99 for the linear regression part of the analysis. Two family practice residents performed all chart reviews as part of the research component of their curriculum. The explanatory variables that appear in the final model were the only variables chosen to be analysed: their selection was based primarily on literature review, since effects of parity, age, IUD type and local anaesthetic on IUD insertions have all been studied. We included previous abortion status as an explanatory variable because it obviously entails manipulation of the cervix and was readily available for review. For our purposes, we defined parity as either having given birth (parous) or never having given birth (nulliparous) to children. Thus it was a dichotomous variable. Statistical analyses were performed by linear multiple regression or analysis of variance (ANOVA) in SPSS (IBM Corporation, New York, NY, USA). Categorical variables were analysed with chi square $\left(\chi^{2}\right)$ tests.

\section{RESULTS}

Of the files reviewed, 354/505 files met the inclusion criteria for the linear regression analysis. The exclusions were 84 IUD insertions at surgical abortion, 41 files missing insertion pain rating, nine files missing parity and 17 files missing local anaesthetic information. Since one cannot assume that pain ratings were missing at random, a comparison of files missing this variable to complete charts was performed via ANOVA or $\chi^{2}$ tests. There were no significant differences in age, parity, rate of cervical anaesthetic or IUD type. One family physician performed $84 \%$ of insertions. Eighty percent were simple IUD insertions not associated with other gynaecological care; $14 \%$ were delayed insertions at least 2 weeks after surgical therapeutic abortion and $6 \%$ were insertions after medical abortion. The average age at IUD insertion was 31 (range 16-51) years. Thirty-seven percent of patients were nulliparous and 63\% were parous (vaginal or Caesarean birth not specified). The IUD was either the levonorgestrel intrauterine system (IUS) $(45 \%)$ or a copper-containing IUD (primarily a CuT380) (54\%) (six uncoded cases).

\section{Procedure description}

Clinic staff recommended that women take ibuprofen $800 \mathrm{mg} 1$ hour prior to the scheduled procedure. Some $95 \%$ of patients did so. No other medication was advised or given. Misoprostol was not used. Bimanual examination was performed before each insertion. The cervix was then visualised through a bivalve speculum. In most cases (86\%) the cervix was then grasped with a single or multi-toothed (atraumatic) tenaculum. The uterus was then sounded with a thin standard metal sound in $67 \%$ of cases. In $10 \%$ of insertions additional instrumentation was required: either an os finder, or cervical dilators or both. Overall, $8 \%$ of insertions were coded as difficult in some manner. A transvaginal ultrasound position check was performed after each insertion. Adverse patient reactions occurred in 4\% of insertions: primarily mild vasovagal reactions and painful cramping. Oxygen was used once during a prolonged vasovagal reaction, although this is not standard treatment. There were no uterine perforations and no insertion failures.

Local anaesthesia was offered routinely to all women. Forty-six percent of women declined. Average dosing was 3.6 (range 1-15) ml of $1 \%$ lidocaine in a 3 or $5 \mathrm{ml}$ syringe injected intracervically 
with a 25 gauge needle. Of those who received cervical anaesthetic, 91\% received 3-5 $\mathrm{ml}$ of lidocaine and only $2 \%$ percent received more than $5 \mathrm{ml}$. No information was recorded on location of the block, although it was office custom to inject anaesthetic initially at the 12 o'clock cervical position before placement of the tenaculum, with additional blocks at the 3 and 9 o'clock positions as necessary. Benzocaine $20 \% \mathrm{w} / \mathrm{w}$ spray was also used occasionally (five cases).

\section{Main findings}

Multiple linear regression analysis of the effects of parity, use of local anaesthetic, age, IUD type and recent abortion status on insertion tolerability was performed (Table 1). The assumptions of this model were verified. Explanatory variables had no colinearity or significant interactions. In the linear regression analysis there were significant effects of local anaesthesia and parity on insertion pain. Nulliparous women reported significantly more pain than did parous women (mean pain rating 2.8 vs $1.7 / 10: t=-2.9 ; p=0.003,95 \%$ CI -1.32 to -0.26 ) (Figure 1). Cervical anaesthesia produced significantly more pain than did no cervical block (2.7 vs 1.4/10: $t=3.9 ; p=0.000,95 \%$ CI 0.50-1.50) (Figure 2). Age, IUD type and recent abortion status did not significantly impact insertion tolerability.

Adverse IUD insertion events are listed in Table 2. The overall rate was $13.9 \%$. The difference in adverse events between nulliparous and parous women did not reach significance (24/127 nulliparous, 25/221 parous; $p=0.05$, Pearson $\chi^{2}=3.84$ ), although there was a trend towards more adverse insertion events amongst nulliparous women. No significant difference in age was found when comparing those with and without insertion adverse events (ANOVA $F=0.008$; $p=0.93$, 95\% CI 30.0-31.6).

All patients were asked to attend for follow-up at the clinic of IUD insertion (Table 3) and 171 women did so. Average time to follow up was 62 (range 3-491, median 37) days. Women who requested IUD removal because they desired pregnancy were excluded from analysis because we specifically wanted to analyse problems in IUD users that led to discontinuation. No significant difference in IUD expulsion/ removal rates by parity was found (12/62 nulliparous vs $19 / 109$ parous; $p=0.754$, Pearson $\left.\chi^{2}=0.099\right)$. The

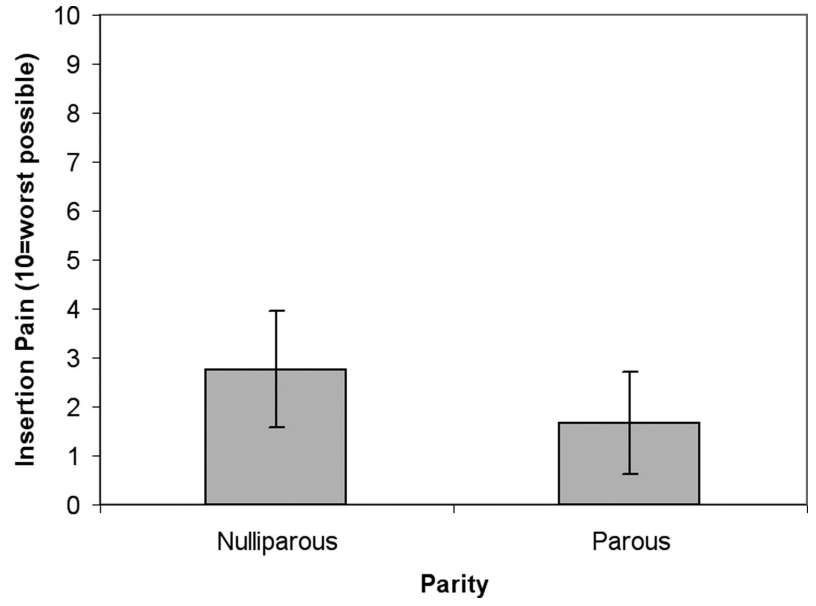

Figure 1 The effect of parity on intrauterine device insertion pain (variance expressed as standard error).

most common reasons for IUD removal in both groups were pain or menstrual irregularities.

\section{DISCUSSION}

Several findings in this study translate into useful clinical information with the potential to improve patientcentred care. First, overall tolerability of IUD insertion is good. Regardless of parity status, the average insertion pain rating was 2.1/10. This finding is corroborated by other studies investigating insertion pain with similar 0-10 visual, rather than verbal, analogue pain rating scales, with reported average insertion pain ratings from 1 to $3.5 / 10 .^{8}{ }^{18}$ Of note, higher pain ratings have also been reported with different visual analogue scales $(55-65 / 100) .{ }^{6}{ }^{19}$ Obviously more work is needed to provide reliable pain ratings for IUD insertions. Women considering an IUD often ask how painful the insertion is, and having an objective, numerical description to pass along is easily comprehensible to patients.

In terms of specific study objectives, we found statistically that significantly more insertion discomfort was experienced by nulliparous women. This finding is in line with other published studies to date. ${ }^{7} 817$ There is wide variability, however, in how insertion pain is defined and operationalised. For instance, one study showing significantly more insertion pain in nulliparous women only recorded insertions as painful if the pain was 'severe', and did not specify how the severity was defined. ${ }^{7}$ A standardised approach, taking into

Table 1 Multiple linear regression analysis of intrauterine device insertion tolerability

\begin{tabular}{lccrrr}
\hline Variable & Coefficient $(\boldsymbol{\beta})$ & SE & 95\% Cl & $\boldsymbol{t}$ & \multicolumn{1}{c}{$\boldsymbol{\boldsymbol { t }}$} \\
\hline Age & 0.005 & 0.016 & $-0.031-0.034$ & 0.092 & 0.003 \\
Parity & -0.168 & 0.270 & $-1.325-0.264$ & -2.944 & 3.929 \\
Local anaesthetic & 0.218 & 0.255 & $0.500-1.501$ & 0.000 \\
Recent abortion & 0.021 & 0.171 & $-0.270-0.405$ & 0.395 & 0.693 \\
IUD type & -0.003 & 0.244 & $-0.496-0.465$ & -0.062 & 0.950 \\
\hline
\end{tabular}

$\mathrm{Cl}$, confidence interval; IUD, intrauterine device; SE, standard error. 


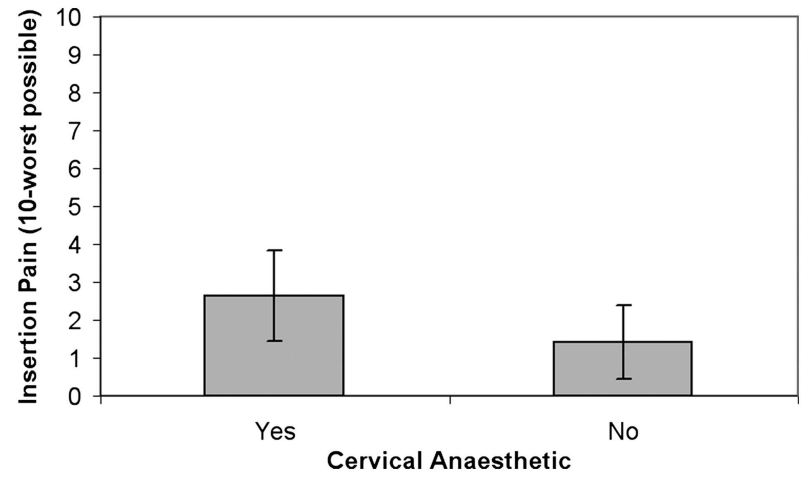

Figure 2 The effect of cervical anaesthesia on intrauterine device insertion pain (variance expressed as standard error).

account clinical relevance, would be helpful to further elucidate this question of differences in insertion pain by parity. When one examines our actual difference in pain scores, a difference between averages of 1.1/10, it is questionable that the statistical difference by parity translates into a clinically important difference that would actually deter one from offering an IUD to a nulliparous woman.

Surprisingly, cervical anaesthesia was related to greater insertion pain: a main effect independent of parity, age, IUD type and previous abortion status. Although the average difference was small, there appears to be no other benefit of administration of local anaesthetic that might offset this increase in pain. This finding is not out of line with several other recent studies showing no reduction in IUD insertion pain with either topical or paracervical lidocaine, but does go against the only other published work on injected intracervical lidocaine. ${ }^{16} 18$ 20-22 The current finding is an important step in providing caregivers with evidence against a standard part of many IUD insertions. In this retrospective study, however, women who accepted or declined local anaesthesia may have differed in consistent ways, such as anxiety level or fear of the procedure, with the potential that such a difference could confound results. A prospective, randomised controlled design would of course be necessary to confirm the causal effects of locally infiltrated anaesthetic.

This study did not find a significant difference in IUD insertion adverse events by parity, although one

Table 2 Adverse intrauterine device insertion events by parity

\begin{tabular}{lcc}
\hline Adverse reaction & $\begin{array}{l}\text { Nulliparous } \\
{[\boldsymbol{n}(\%)](\boldsymbol{n}=\mathbf{1 2 7})}\end{array}$ & $\begin{array}{l}\text { Parous } \\
{[\boldsymbol{n}(\%)](\boldsymbol{n}=\mathbf{2 2 1})}\end{array}$ \\
\hline Vagal reaction* & $4(3.1)$ & $5(2.3)$ \\
Severe pain & $1(0.8)$ & $2(0.9)$ \\
Other adverse reactiont & $1(0.8)$ & $1(0.5)$ \\
Difficult insertion $\neq$ & $18(14.2)$ & $17(7.7)$ \\
Total & $24(18.9)$ & $25(11.3)$ \\
\hline
\end{tabular}

${ }^{*}$ Nausea, light-headedness, sweating.

†Vaginal bleeding, extreme anxiety.

$\ddagger$ Additional instrumentation required: os finders, cervical dilators or both.

IUD, intrauterine device. could argue for a trend towards more difficulties amongst nulliparous woman. Of note, no 'difficult' insertions resulted in failures. They simply required additional instrumentation: os finders or cervical dilators during $14 \%$ of nulliparous insertions. Having such instruments easily available might be a consideration for those performing frequent IUD insertions in nulliparous women. Our rate of adverse insertion events is higher than in other reported studies, a difference probably accounted for by the fact that 'difficult' insertions, as judged by the objective need for additional instrumentation, were included as an insertion complication for the purpose of this analysis. Only more serious adverse events, such as perforation and failure, were classified as difficult in other studies. ${ }^{7}$ The purpose of our study's broader definition of an insertion adverse event was to more accurately capture the experience of the physician at insertion, because not all difficult insertions end in negative outcomes. This study was underpowered to reliably assess uncommon insertion events such as vasovagal reaction. One other study with a larger dataset has found that nulliparous women are more likely to experience a vasovagal event. ${ }^{15}$ Another larger data set also reported more failed insertions, cervical problems and bradycardia in women who had never had a vaginal delivery. ${ }^{17}$

Although our follow-up data are incomplete in that data were available for less than half of the IUD insertions analysed, our findings are in line with other similar retrospective reviews, which also did not find a difference in expulsions or removals by parity. ${ }^{11} 1323$ Comparatively, our expulsion rate for nulliparous women is very close to at least one other published study (6.5\% vs $7 \%$, respectively). ${ }^{6}$ Our continuation rates, however, are certainly higher at 80 $83 \%$ than the $55-67 \%$ cited elsewhere, suggesting that a significant number of discontinuations may have been missed. ${ }^{910}{ }^{13}$ Yet at least part of this difference is due to the fact that we excluded IUD removals for women desiring pregnancy because we felt it inappropriate to include this as a follow-up complication of IUD use.

One limitation of the current dataset is that the physician who completed most of the IUD insertions

Table 3 Intrauterine device post-insertion complications by parity

\begin{tabular}{lcc}
\hline Post-insertion complications & Nulliparous & Parous \\
\hline All IUD insertions $(n)$ & 148 & 265 \\
Follow-up appointment $[n(\%)]$ & $62(42)$ & $109(41)$ \\
IUD expulsion or removal $(n)$ & 12 & 19 \\
$\quad$ All insertions (\%) & 8.1 & 7.2 \\
Only those with follow-up (\%) & 19.4 & 17.4 \\
Expulsion rate ( $n$ ) & 4 & 3 \\
All insertions (\%) & 2.7 & 1.1 \\
Only those with follow-up (\%) & 6.5 & 2.8 \\
\hline
\end{tabular}

IUD, intrauterine device. 
does so at a high volume of several insertions per week. This might be reflective of a reproductive health clinic in North America but most general practitioners perform insertions much less often. Thus rates of adverse events in this study might not be generalisable to all other practitioners inserting IUDs. Another limitation of the current study is that a sizeable number of charts reviewed were missing a rating for insertion pain and were therefore excluded from analysis. Statistical comparisons failed to find any difference between insertions missing this data point and those that were complete in terms of most of the main variables that were assessed. Despite these precautions, it is possible that these pain data were not missing at random and that important information thus may not be represented in the current analysis.

Despite these shortcomings, our data suggest that the practice of local anaesthesia at IUD insertion causes more, rather than less, pain. Additionally, it shows that women of all reproductive ages seem to tolerate the IUD similarly. It also sheds further light on the debate around IUD use in nulliparous women. On the one hand, the current results add to the growing support for IUDs as safe and well-tolerated in nulliparous women, without significantly more insertion adverse events or follow-up complications than parous women. On the other hand, this study suggests that some differences across parity do exist. Perhaps the most productive route for future research would be to further elucidate the clinical relevance of any differences, rather than attempting to prove equivalence between nulliparous and parous IUD users.

Funding This study was partially funded by a British Columbia College of Family Physicians Resident Research Award.

\section{Competing interests None.}

Ethics approval Ethical approval was granted by the University of British Columbia's Clinical Research Ethics Board (H07-01087).

Provenance and peer review Not commissioned; externally peer reviewed.

\section{REFERENCES}

1 Farley TM, Rosenberg MJ, Rowe PJ, et al. Intrauterine devices and pelvic inflammatory disease: an international perspective. Lancet 1992;339:785-788.

2 Xiong X, Buekens P, Wollast E. IUD use and the risk of ectopic pregnancy: a meta-analysis of case-control studies. Contraception 1995;52:23-34.

3 Grimes DA. Intrauterine device and upper-genital-tract infection. Lancet 2000;356:1013.

4 Deans EI, Grimes DA. Intrauterine devices for adolescents: a systematic review. Contraception 2009;79:418-423.
5 World Health Organization. Medical Eligibility Criteria for Contraceptive Use. 2008 Update. 2008. http://whqlibdoc.who. int/hq/2008/WHO_RHR_08.19_eng.pdf [accessed 10 February 2013].

6 Brockmeyer A, Kishen M, Webb A. Experience of IUD/IUS insertions and clinical performance in nulliparous women: a pilot study. Eur J Contracept Reprod Health Care 2008;13:248-254.

7 Lassner KJ, Chen CH, Kropsch LA, et al. Comparative study of safety and efficacy of IUD insertions by physicians and nursing personnel in Brazil. Bull Pan Am Health Organ 1995;29:206-215.

8 Hubacher D, Reyes V, Lello S, et al. Pain from copper intrauterine device insertion: randomized trial of prophylactic ibuprofen. Am J Obstet Gynecol 2006;195:1272-1277.

9 Peterson KR, Brooks L, Jacobsen N, et al. Clinical performance of intrauterine devices in nulligravidae: is the length of endometrial cavity of significance. Acta Eur Fertil 1991;22:225-228.

10 Weiner E, Berg AA, Johanson I. Copper intrauterine contraceptive devices in adolescent nulliparae. Br J Obstet Gynaecol 1978;85:204-206.

11 Duenas JL, Albert A, Carrasco F. Intrauterine contraception in nulligravid vs parous women. Contraception 1996;53:23-24.

12 Wildemeersch D, Van Kets H, Vrijens M, et al. Intrauterine contraception in adolescent women. The GyneFix intrauterine implant. Ann NY Acad Sci 1997;816:440-450.

13 Veldhuis HM, Vos AG, Lagro-Janssen AL. Complications of the intrauterine device in nulliparous and parous women. Eur J Gen Pract 2004;10:82-87.

14 Alton TM, Brock GN, Yang D, et al. Retrospective review of intrauterine device in adolescent and young women. J Pediatr Adolesc Gynaecol 2012;25:195-200.

15 Chi IC, Wilkens LR, Siemens AJ, et al. Syncope and other vasovagal reactions at interval insertion of Lippes Loop D - who is most vulnerable? Contraception 1986;33:179-187.

16 Hepburn S. Method of local anesthesia for IUD insertion. Contracept Deliv Syst 1980;1:371-377.

17 Farmer M, Webb A. Intrauterine device insertion-related complications: can they be predicted? J Fam Plann Reprod Health Care 2003;29:227-231.

18 Mohammad-Alizadeh-Charandabi S, Seidi S, Kademi F. Effect of lidocaine gel on pain from copper IUD insertion: a randomized double-blind controlled trial. Indian J Med Sci 2010;64;349-355.

19 Edelman AB, Schaefer E, Olson A, et al. Effects of prophylactic misoprostol administration prior to intrauterine device insertion in nulliparous women. Contraception 2011;84:234-239.

20 Mody SK, Kiley J, Rademaker A, et al. Pain control for intrauterine device insertion: a randomized trial of $1 \%$ lidocaine paracervical block. Contraception 2012;86:704-709.

21 McNicholas CP, Madden T, Zhao Q, et al. Cervical lidocaine for IUD insertional pain: a randomized controlled trial. Am J Obstet Gynecol 2012;207:384.e1-384.e6.

22 Maguire K, Davis A, Rosario Tejeda L, et al. Intracervical lidocaine gel for intrauterine device insertion: a randomized controlled trial. Contraception 2012;86:214-219.

23 Behringer T, Reeves MF, Rossiter B, et al. Duration of use of a levonorgestrel IUS amongst nulliparous and adolescent women. Contraception 2011;84:e5-e10. 